\title{
Guanidinoacetate-Creatine Supplementation Improves Functional Performance and Muscle and Brain Bioenergetics in the Elderly: A Pilot Study
}

\author{
Vesna Sepera, b Darinka Korovljev ${ }^{\mathrm{a}}$ Nikola Todorovic $^{\mathrm{a}}$ Valdemar Stajer $^{\mathrm{a}}$ \\ Jelena Ostojic ${ }^{a, c}$ Nebojsa Nesic ${ }^{b}$ Sergej M. Ostojic ${ }^{a}$ \\ ${ }^{a}$ Applied Bioenergetics Lab, Faculty of Sport and PE, University of Novi Sad, Novi Sad, Serbia; ${ }^{6}$ Physical Therapy \\ Studies, College of Applied Sciences Lavoslav Ruzicka, Vukovar, Croatia; 'Department of Physiology, Faculty of \\ Medicine, University of Novi Sad, Novi Sad, Serbia
}

\section{Dear Editor,}

During the past 2 decades or so, the scientific community continues to scrutinize various nutritional strategies aimed to attenuate aging-associated impairments in functional abilities and cognition. From individual micronutrients and nonnutrients to complete dietary interventions, previous studies identified several food components that might support healthy aging and reduce agerelated disorders [1]. One purported candidate is creatine, which has been shown to have promise on improving disorders associated with skeletal muscle and brain aging [2]. However, several factors seem to limit the effectiveness of dietary creatine as a therapeutic intervention in the elderly, including restraints in transport-mediated kinetics or finite storage capacity [3]. Guanidinoacetate (GAA, also known as guanidinoacetic acid or glycocyamine) is a naturally occurring creatine precursor with advanced transportability and an innovative dietary supplement that might increase the rate of creatine turnover. GAA-creatine mixture was found to outperform creatine in boosting brain and muscle performance in adult men and women [4], yet whether this mixture facilitates tissue bioenergetics, and cognitive and functional performance in the elderly remains unaddressed so far. The objective of this pilot trial was to evaluate the effects of 8-week GAA-creatine supplementation versus placebo on skeletal muscle and brain creatine levels, cognitive function, functional outcomes, and safety biomarkers in men and women aged 65 years and older.

Twenty-one healthy elderly individuals (age 69.6 \pm 4.9 years, body mass index $27.6 \pm 4.2 \mathrm{~kg} / \mathrm{m}^{2} ; 13$ women) signed informed consent to voluntarily take part in this multicenter randomized, double-blind, placebo-controlled crossover trial. The sample size $(n=21)$ was computed using $\mathrm{G}^{*}$ Power (Mac version 3.1.9.3; Heinrich Heine University, Düsseldorf, Germany), with the effects size fixed at 0.50 , alpha error probability 0.05 , power 0.80 , for 2 supplement groups (GAA-creatine and placebo), and 3 measurements of study outcomes. All participants were randomly assigned to receive a GAA-creatine mixture ( $2 \mathrm{~g}$ /day of GAA and $2 \mathrm{~g} /$ day of creatine) or placebo (inulin) for 8 weeks; the washout period lasted for 4 weeks to prevent any residual effects of interventions across study periods. The participants were asked to refrain from using any other dietary supplements and maintain their usual lifestyle (including diet and physical activity) karger@karger.com

(c) 2021 S. Karger AG, Basel

www.karger.com/anm

Karger ${ }^{\prime \prime}=$
Correspondence to:

Sergej M. Ostojic, sergej.ostojic@ chess.edu.rs 


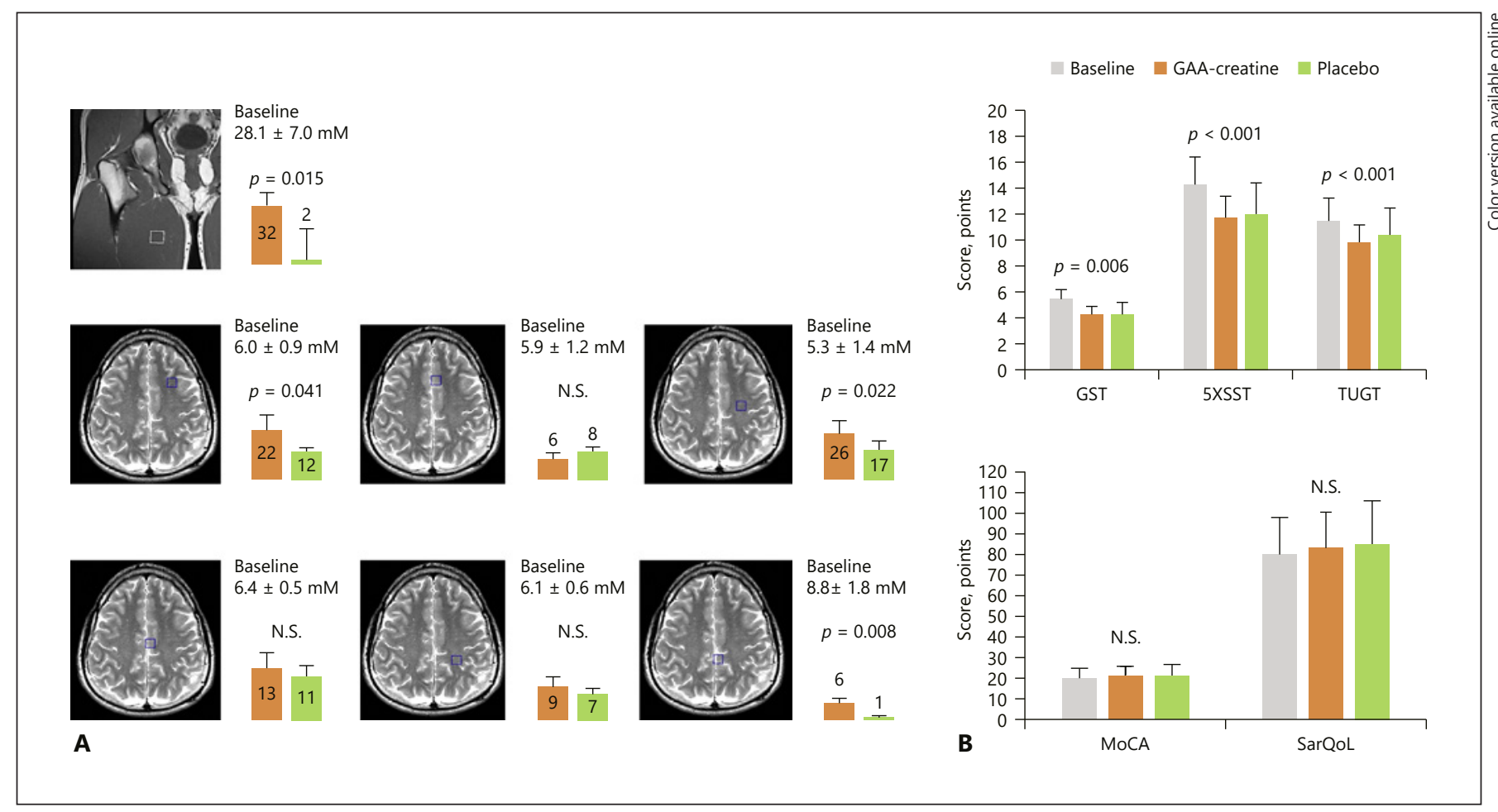

Fig. 1. Baseline creatine levels and percent change after GAA-creatine (orange) and placebo intake (green) across individual locations (marked with a square) in vastus medialis muscle and brain during the trial (A). Changes in 4-m GST, 5-repetition Sit-to-Stand Test (5XSST), TUGT, cognitive function (MoCA), and health-related quality of life (SarQoL) from baseline (gray) to post-admin- istration for each intervention (B). $p$ values were calculated from a 2-way mixed model ANOVA (treatment vs. time interaction) and Friedman's 2-way ANOVA by ranks. N.S., nonsignificant; GST, gait speed test; TUGT, Timed Up and Go Test; GAA, guanidinoacetate. during the study, as monitored by physical activity questionnaires and 3-day food records. The primary outcome (brain creatine levels) and secondary outcomes (muscle creatine levels, cognitive performance, sarcopenia quality-of-life score, functional mobility, and safety biomarkers) were assessed at baseline (pre-intervention) and 8 -week follow-up. Tissue creatine levels in 6 brain regions of interest, and vastus medialis muscle were evaluated with $1.5 \mathrm{~T}$ proton magnetic resonance spectroscopy (MRS) (Avanto Scanner Siemens, Germany), as previously described [4], with intra- and inter-subject CV values of $\leq 10 \%$ and $\leq 15 \%$, respectively. Cognitive function was determined via Montreal Cognitive Assessment (MoCA) test, while the health-related quality of life was evaluated with a sarcopenia-specific questionnaire (SarQoL). The functional performance included a 4-m gait speed test, 5-repetition Sit-to-Stand Test (5XSST), and Timed Up and Go Test (TUGT) that measure mobility, functional strength, and fall risk in older adults. Safety biomarkers included clinical enzymes, total protein, blood urea nitrogen, creatinine, and C-reactive protein. The study was carried out at FSPE Applied Bioenergetics Lab and College of Applied Sciences between June and December 2020, and the local IRB at the University of Novi Sad approved the study protocol (\# 46-06-01/2020-1-CREGAAS). All procedures were performed in accordance with the Declaration of Helsinki, and the trial was registered at ClinicalTrials.gov (NCT04652921). A 2-way ANOVA with repeated measures or Friedman's 2-way ANOVA by ranks was used to establish if any significant differences existed between participants' responses over time of intervention (baseline vs. post-administration), with the intervention (GAA-creatine or placebo) included as a between-subjects factor; post hoc analyses were employed to identify the differences between individual sample pairs.

All participants completed the trial, with no participant reporting any side effects of either treatment. MRS revealed that GAA-creatine significantly increased brain and muscle creatine levels in individual regions of interest 
from baseline to 8-week follow-up, as compared to placebo $(p<0.05)$ (Fig. 1). The mixture was significantly superior to placebo to improve gait speed test, 5XSST, and TUGT performance at post-administration $(p<0.01)$, while no differences were found between interventions for MoCA and SarQoL scores $(p>0.05)$. No differences were observed between treatment groups for safety biomarkers $(p>0.05)$, except for increased serum creatinine after GAA-creatine intake comparing to placebo (19.3\% vs. $14.3 \%$; $p<0.05)$; no participants experienced clinically significant creatinine rise $(>114.9 \mu \mathrm{mol} / \mathrm{L}$ for men, and $>97.2 \mu \mathrm{mol} / \mathrm{L}$ for women).

In this randomized controlled trial conducted in older men and women, co-administration of GAA and creatine resulted in significantly improved concentrations of the brain and muscle creatine, and functional mobility outcomes at 8-week follow-up compared with a placebo intervention. Our results largely confirm the findings from a recent human trial that demonstrated improved muscular performance and tissue bioenergetics with supplemental GAA-creatine when compared with creatine alone [4]. Interestingly, the mixture-driven augmentation of creatine levels in the skeletal muscle (also across the brain) in the elderly appears to be superior compared to younger counterparts in a previous study. This implies a possible age-specific response to the treatment, with the elderly may be more sensitive to GAA-creatine intervention due to lower levels of tissue creatine and/or favorable transport kinetics. A 3T phosphorus MRS ( $\left.{ }^{31} \mathrm{P}-\mathrm{MRS}\right)$ is generally accepted as a gold standard for evaluating sources of metabolic energy, with increased sensitivity to changes [5]. We used here $1.5 \mathrm{~T}^{1} \mathrm{H}$-MRS yet no significant differences in the metabolite ratios were found between the 2 field strengths in the past studies [6], with ${ }^{1} \mathrm{H}-\mathrm{MRS}$ remains the major clinically applied method in neurospectroscopy as it provides relevant information on metabolic active compounds [5]. Although more studies are needed to corroborate our preliminary findings, GAA-creatine might be recognized as a nutritional intervention with the potential to tackle compromised tissue bioenergetics and functional impairments associated with aging.

\section{Statement of Ethics}

The study was conducted according to the guidelines of the Declaration of Helsinki. The ethical approval to conduct the study was granted by the local IRB at the University of Novi Sad (\# 46-06-01/2020-1-CREGAAS). Informed consent was obtained from all participants.

\section{Conflict of Interest Statement}

V.S., D.K., N.T., V.S., J.O., and N.N. declare no conflicts of interest. S.M.O. serves as a member of the Scientific Advisory Board on creatine in health and medicine (AlzChem LLC). S.M.O. owns patent "Sports Supplements Based on Liquid Creatine" at European Patent Office (WO2019150323 A1), and active patent application "Synergistic Creatine" at UK Intellectual Property Office (GB2012773.4). S.M.O. has served as a speaker at Abbott Nutrition, a consultant of Allied Beverages Adriatic and IMLEK, and an advisory board member for the University of Novi Sad School of Medicine, and has received research funding related to creatine from the Serbian Ministry of Education, Science, and Technological Development; Provincial Secretariat for Higher Education and Scientific Research; AlzChem GmbH; KW Pfannenschmidt $\mathrm{GmbH}$; ThermoLife International LLC; and Monster Energy Company. S.M.O. is an employee of the University of Novi Sad and does not own stocks and shares in any organization. The funders had no role in the design of the study; the collection, analyses, or interpretation of data; the writing of the manuscript; or the decision to publish the results.

\section{Funding Sources}

This research received no external funding.

\section{Author Contributions}

V.S.: conducted research, analyzed data, performed statistical analysis, wrote paper draft, and revised the paper. D.K.: conducted research, analyzed data, performed statistical analysis, wrote paper draft, and revised the paper. N.T.: conducted research, analyzed data, performed statistical analysis, wrote paper draft, and revised the paper. V.S.: conducted research; analyzed data, performed statistical analysis, and revised the paper. J.O.: conducted research, analyzed data, performed statistical analysis, and revised the paper. N.N.: conducted research, analyzed data, performed statistical analysis, and revised the paper. S.M.O.: designed research (project conception, development of overall research plan, and study oversight), analyzed data, performed statistical analysis, wrote paper draft, and had primary responsibility for final content. All authors read and approved the final manuscript.

\section{Data Availability Statement}

Data described in the manuscript will be made available upon request. 


\section{References}

1 Meydani M. Nutrition interventions in aging and age-associated disease. Ann N Y Acad Sci. 2001;928:226-35.

2 Candow DG, Chilibeck PD, Forbes SC. Creatine supplementation and aging musculoskeletal health. Endocrine. 2014;45(3):35461.
3 Ostojic SM. Overcoming restraints of dietary creatine. Trends Food Sci Technol. 2020;100: 246-7.

4 Semeredi S, Stajer V, Ostojic J, Vranes M, Ostojic SM. Guanidinoacetic acid with creatine compared with creatine alone for tissue creatine content, hyperhomocysteinemia, and exercise performance: a randomized, doubleblind superiority trial. Nutrition. 2019;57: 162-6.
5 Burtscher IM, Holtås S. Proton MR spectroscopy in clinical routine. J Magn Reson Imaging. 2001;13:560-7.

6 Kim JH, Chang KH, Na DG, Song IC, Kim SJ, Kwon BJ, et al. Comparison of $1.5 \mathrm{~T}$ and $3 \mathrm{~T} 1 \mathrm{H}$ MR spectroscopy for human brain tumors. Korean J Radiol. 2006 Jul-Sep;7(3):156-61. 